\title{
From Monolithics to Tethers to Freeflyers: The Spectrum of Large Aperture Sensing from Space
}

\author{
Jesse Leitner and David Quinn \\ NASA/Goddard Space Flight Center, GN\&C Division \\ Jesse.Leitner@gsfc.nasa.gov, phone: 301-286-2630
}

\begin{abstract}
As part of NASA's endeavor to push the envelope and go where we have never been before, the Space Science Enterprise has laid out a vision which includes several missions that revolutionize the collection of scientific data from space. Many of the missions designed to meet the objectives of these programs depend heavily on the ability to perform space-based interferometry, which has recently become a rapidly growing field of investigation for both the scientific and engineering communities. While scientists are faced with the challenges of designing high fidelity optical systems capable of making detailed observations, engineers wrestle with the problem of providing space-based platforms that can permit this data gathering to occur. Observational data gathering is desired at a variety of spectral wavelengths and resolutions, calling for interferometers with a range of baseline requirements. Approaches to configuration design are as varied as the missions themselves from large monolithic spacecraft to multiple free-flying small spacecraft and everything in between. As will be discussed, no one approach provides a 'panacea' of solutions rather each has its place in terms of the mission requirements. The purpose here is to identify the advantages and disadvantages of the various approaches, to discuss the driving factors in design selection and determine the relative range of applicability of each design approach.
\end{abstract}

\section{INTRODUCTION}

It is well-known that the ability to obtain fringe patterns from an interferometer is enabled by precise knowledge and control of all the elements in the optical path. This is particularly challenging for interferometry at short wavelengths and where the angular resolution is extremely precise (i.e., the baseline is large) and the baseline is 10-15 orders of magnitude larger than the requirement for precise positioning. For an interferometer on an optical bench, the table is relatively rigid since, over the short length of the table, flexible body motions of the massive table are very small compared to the wavelengths being measured, therefore flexible modes occur at very high frequencies. In this case, the flexible body dynamics of the table are not relevant and so may be ignored. However, if the elements of the interferometer are required to be at such a great distance apart that the structure to which it is mounted cannot be considered rigid, the flexible body dynamics can no longer be ignored. This is particularly the case in space where the baselines are larger, the mass is constrained, and there is no direct manual ability to tweak actuators to obtain the fringe patterns. The knee in the curve seems to occur at the point where the expected range of motion of the critical points on the connecting structure (the table in our example) grows beyond the allowable range of motion. When the motions in the natural disturbance environment are greater than the allowable tolerances, controls are introduced to contain the motions within the allowable tolerances. Control is applied through the use of actuators which themselves have a range (and resolution) of operation. Obviously, the control range of any actuator needs to be wider than the range of motions the actuator is expected to control. When extremely high precision is required over a wide dynamic range, multiple actuators may be employed with overlapping control ranges and resolutions that allow coverage of the wide dynamic range and yet provide for the desired precision. Last, but not least, even in the quiet 
space environment, some of the worst enemies of the engineer are his own doing. For example, the mere existence of actuators, including optical path delay lines, can be culprits for the instigation of disturbances. This fact must considered before selecting a concept which requires internal active controls.

\section{MEASUREMENT AND ESTIMATION}

It is important to note that generally speaking, a sensor or metrology system does not provide the specific measurements needed for the performance objective. Henceforth, the measurements must be transformed into performance variables of interest. If these performance variables are dynamic, requiring real-time control in the vicinity of disturbances and modal vibrations, then the measurements must pass through a filter that includes (at the very least) all structural modes observable at the measurement variables. [1, Kailath, p. 120-175]. In basic terms, the context of the measurement is very often as important as the measurement itself. If a given measurement is to have meaning, an understanding and model of the physical system used to make the measurement must be included in the statement of the problem. To accurately model the physical system, all of the relevant dynamics of the system must therefore be represented. Deciding what dynamics are relevant and need to be modeled is the point at which the real trouble begins.

For example, it is very often assumed that if an accurate enough range measurement between two elements of an interferometer is made, that the dynamics of the intervening structure is irrelevant. This is not generally the case. Where the connecting structure is rigid and motion is permitted only in the direction of the range measurement, this is true since all relative motion is constrained by the rigidity and captured by the measurement. However, knowing even the exact distance between two points on the surface of a flexible membrane or structure is not sufficient to measure the motions among all of the degrees of freedom introduced by the membrane or structure itself, much less control them. A model of the flexible body and an understanding of the how the disturbance source is applied is required to determine all of the quantities of interest.

Another example is the Global Positioning System, or GPS, in which raw measurements (ranges or carrier phases) are used to compute the location of the user. Even if perfect, these raw measurements are themselves useless without a dynamic model of the vehicle with the collection antenna(e) and the GPS constellation. Since during the course of taking the measurement, the "connecting body" has moved and coordinate frames have changed (even if by a small amount), the relevant dynamics must be propagated to account for those changes. The better the model, the better the estimate; likewise the tighter the tolerances, the more important this part becomes. If a simple pseudo-inverse transform is performed, a crude navigation fix may be estimated from each observation independently (the so-called Single Point Solution). However, improve the model by including the dynamics of the GPS constellation and a vastly improved estimate results. In this case, the range measurements are taken in the context of the vehicle dynamics and the motion of the GPS constellation to determine the user's location. The bottom line is that a raw measurement is not generally sufficient directly from the sensor, in particular when a highly precise measurement is required. A model of the system in which the measurement is made is required in order to determine any quantities outside of the raw measured signal (which is rarely the desired quantity), because of motions and noise in the system. 


\section{THE SPECTRUM OF LARGE APERTURE IMAGING APPROACHES}

In this section we characterize and put in proper context the approaches for large aperture sensing from space. References [2, Ultralite prog. Ov., 3, Assessment of Large Aperture ...] detail some of the relevant trades for sparse aperture systems, primarily focused on contiguous (i.e., not free-flying) sub-apertures. In this paper we generalize, more qualitatively, with a focus on comparing the contiguous to free-flying approaches.

\section{Monolithic Aperture}

The monolithic mirror approach reached its zenith in the Hubble Space Telescope (HST), with its 2.5 meter primary aperture, shown in Figure 1a. The aperture (along with the additional overhead of the spacecraft) was designed to be the maximum diameter that could be fit into the Shuttle cargo bay. It also happens to be roughly the maximum size for the Titan IV launch vehicle fairing. The monolithic mirror is the extreme case in which the system is bound to one vehicle with the best possible signal-to-noise ratio and optical performance (point-spread function), but the least flexibility. Improved resolution requirements demand a larger aperture beyond the ability for the current generation of launch vehicles to get into orbit as a single solid collecting surface. Quite clearly if the science requirements can be met by this approach, it is the obvious choice. Most importantly the instrument can be verified on the ground to the greatest extent among the large aperture approaches addressed herein.
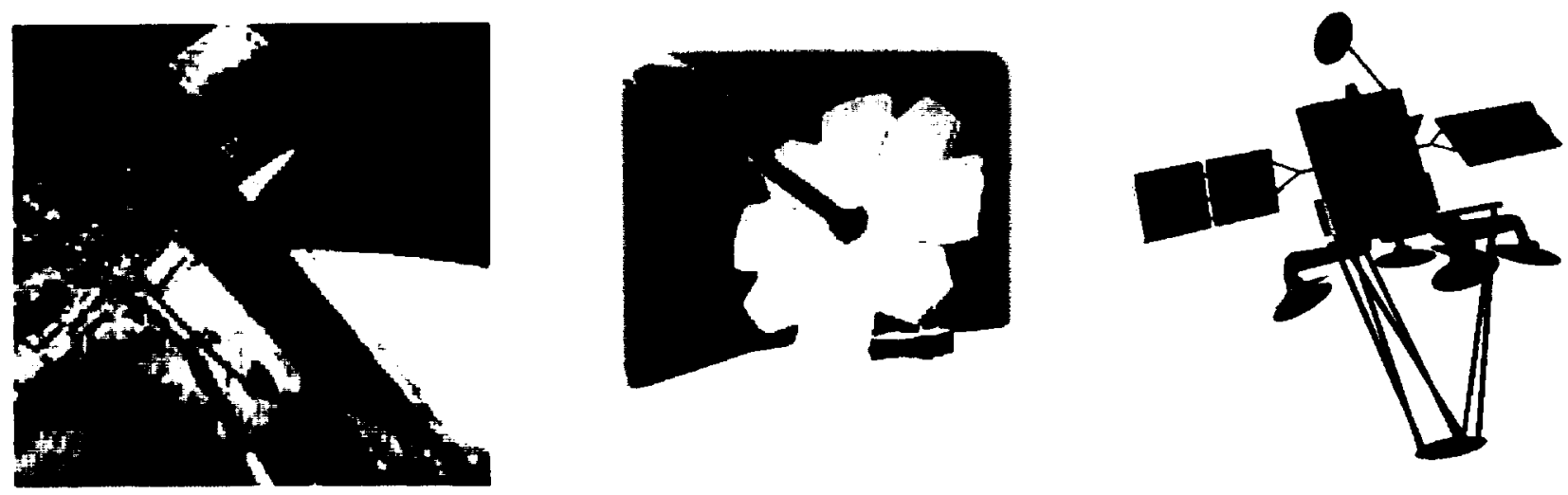

Figure 1: apertures: (a) Monolithic (HST), (b) deployable filled (NGST), (c) deployable sparse

\section{Deployable Filled Aperture}

The next step beyond HST within NASA's Origins Program in the Space Science Enterprise is the Next Generation Space Telescope (NGST), one concept of which is shown in Figure lb, which has a requirement for vastly finer resolution than its predecessor. Since it was clear that there was little chance of vastly increasing the size of launch vehicle capacity, there was no doubt that a deployable aperture offered the only means of attaining the increased resolution with a single vehicle. Henceforth, the initial trades for NGST were whether the deployed aperture should be sparse or filled. In the first phase of the NGST program there were many trades between sparse and filled apertures. The filled aperture concepts won out due, in part, to a much lower perceived risk, the greater achievable signal-to-noise ratio, and the assertion that when primary segments are contiguous, more options exist for measuring and correcting figure errors, such as edge detection schemes. 


\section{Deployable Sparse Aperture}

When the image source is photon rich and the required maximum baseline is not extremely large (less than 10 meters), another viable approach to forming the effective large aperture is through sparsely populated segments. This configuration is driven by either a discrete requirement for particular baselines or an optimization over the modulation transfer function. As the maximum baseline grows, this concept effectively becomes the long boom concept. Figure lc shows a concept analyzed in ref $[2$,ultralite] at AFRL in the UltraLITE project.
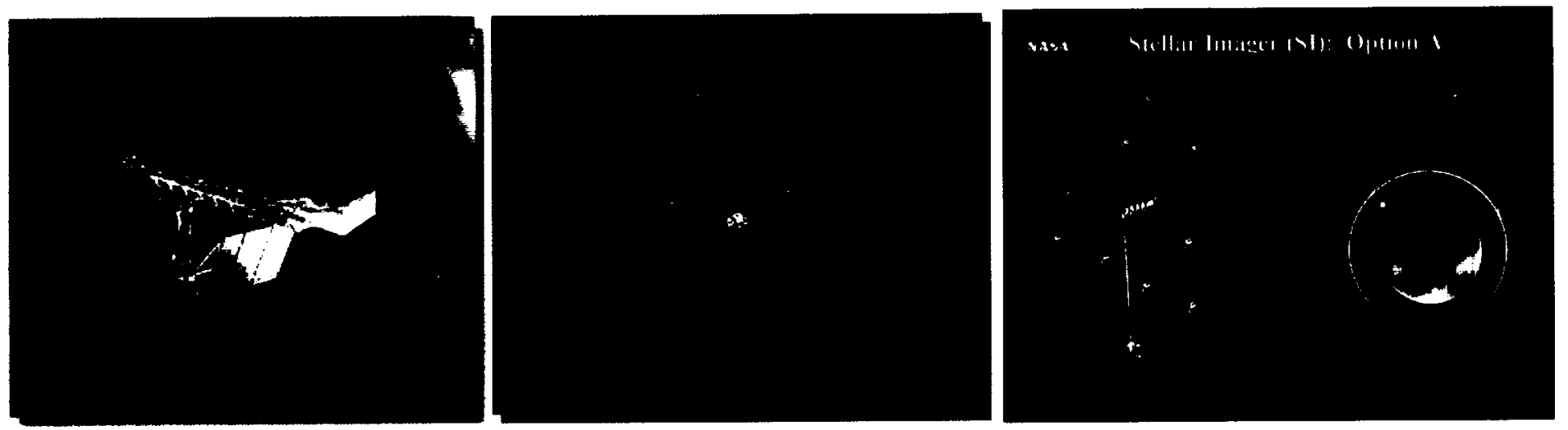

Figure 2: apertures: (a) Long-boom (SIM), (b) tethered (SPECS), (c) free-flyers (Stellar Imager)

Significant effort to date has gone into the exploration of these concepts for the UltraLITE program and related follow-on activities, as well as at the Naval Research Lab and NASA (as part of the now defunct NEXUS project associated with NGST).

\section{Sparse Apertures Over Long Booms}

Expanding the sparse aperture concept further, engineers and scientists began to propose distributing the sparse apertures over long, lightweight, deployable booms. As a precursor to realizing these concepts, over a period of about 20 years, both NASA and the Strategic Defense Initiative Office (SDIO) have funded a great deal of work in the control of large structures much of which was focused on enabling large aperture imaging from space. The extreme challenges of long baseline optical path length control became evident from a vast pool of research in this area and, among others, the following general observations were made:

- The control of distant points along a flexible structure to any significant precision requires substantial control of the relative degrees of freedom joining them. This observation gave rise to a desire to investigate formation flying as an alternative approach when the baselines become very large, hence eliminating all of the internal degrees of freedom.

- The existence of "microdynamics" within large structures began to become clear. This phenomenon of small scale motions excited by thermal pings and pops in the structure and 
minor internal and external disturbances makes its presence due to nonlinear effects caused by imprecise hinges, latches, and joints, etc. While these are small-scale effects relative to a large structure, they are large-scale effects relative to precise positioning requirements and they can't be ignored for short wavelength (visible and below) optical phasing problems. The ways to deal with such problems are (1) through precise hinges, latches, and joints which for a large structure such as a long boom, the challenges are immense or (2) significant internal active control through "smart" members embedded throughout.

In more recent years, the Jet Propulsion Lab (JPL) has been developing and proving out the technologies required for enabling a large-baseline Michelson interferometer in space known as the Space Interferometry Mission, or SIM, shown in Figure 2a. The research and development activity in support of SIM has spawned some critical facilities for world-class development and evaluation of high-precision (pico-meter class) metrology on large flexible space structures [4, SIM1,5, SIM2]. Another practical example of the limitation of long booms for creating the baseline over long booms is in the use of GPS for attitude determination. This is a form of long wavelength interferometry where the attitude of a vehicle is estimated from the relative positions of GPS antennae at multiple positions along the vehicle. The larger the baseline of separation, the more precise the measurement of attitude achievable. One meter of baseline provides approximately one half degree of accuracy in estimation of attitude. Much greater baselines would produce much finer measurements. However, even in the long L-band wavelengths of the GPS system, the requirements for interferometry are extremely stringent. Henceforth, while there may be an appealing sound to placing GPS antennae on aircraft wingtips or on long, telescoping, lightweight booms on spacecraft, in reality, there is a "knee in the curve" just greater than a meter, where the degradation due to structural motions overcomes the advantage of having the additional baseline [6, how, jgcd]. The problem is analogous for interferometry in the shorter wavelengths as well.

\section{Sparse Apertures Connected by Tethers}

When the resolution requirements of a given mission become more and more stringent, the long boom approach becomes overly challenging. In some sense the structure is overly flexible to maintain precision at key locations, while being overly rigid to control the motions at such locations. Furthermore, the uncertainties in a system become extreme due to nonlinearities in the structure, mostly due to the joints and latches. In such instances, tethers can become a viable compromise between the control capability and fuel savings offered by connected systems and the large distances permitted by free flying systems. In fact since off-axis stiffness diminishes with length, a boom of sufficient length can be regarded as a tether. Such is the case with the SPECS (Submillimeter Probe of the Evolution of Cosmic Structure) mission concept proposed as a follow-on to NGST for the 2015 timeframe [7]. High-resolution interferometric imaging requires simultaneous illumination of a differential scene element from the vantage of two or more widely separated observation points. Full scene development requires an appropriately synchronized scan profile of each of the collecting sensors. Such imaging missions have been proposed for free flying distributed spacecraft whose orbits diverge slowly, so as not to require excessive thrusting to reconstitute the system geometry. Nevertheless, the precision required mandates nearly continuous thrusting, which in turn creates the need for complex compensation schemes to enable interferometric fringe-tacking preventing image smear and ultimate loss of resolution. Depending on the source frequency being observed, it may not be suitable to place the sensor in near Earth orbit because of the thermal or particle environment. For example, SPECS requires a sub $4^{\circ} \mathrm{K}$ environment for its instruments, and the Earth-Sun $\mathrm{L}_{2}$ position would be a much more advantageous orbit location for mission of this sort. However, placement of a set of free flying distributed spacecraft about the $\mathrm{L}_{2}$ point would be impossible to sustain for any reasonable time, as 
Earth gravity is no longer the dominant orbit influence. In fact, analyses show that the system would fly apart in less than a day even with more than a fifty-percent propellant mass fraction allocated to each spacecraft. Henceforth, the fuel savings can be substantial with by absorbing the separation energy into tethers, as opposed to continuously firing thrusters.

SPECS is one example of a specific application where it is desirable to maintain a set of distributed sensors relative to a central focal point at a controlled, but variable distance, wherein the focal point is itself moving in a prescribed orbit. The requisite kinematics can best be met through the use of a rotating structure, which provides centrifugal stiffening without the need for reaction propellant. Consideration has been given to deployable structures for each sub-satellite and a detailed analysis performed of two possible configurations employing a tethered implementation [8]. Although some may consider semi-rigid deployable booms to be a preferred implementation, there does not appear today to be any practical solution to developing a 500 meter mast at any reasonable weight and volume, nor is there one forthcoming. Using current technology, a single $500 \mathrm{~m}$ boom when stowed would occupy a compressed length greater than that of the shuttle cargo bay. A tethered system, given all of the obvious concerns, readily overcomes the prohibitive requirements imposed by either a free-flying constellation or the deployable boom solution, and provides the optimum low mass structure with which to accomplish spacebased interferometry and the extraordinary science that it can achieve.

\section{Free Flying Spacecraft in Formation}

The demands of some missions will simply not permit optical elements to be connected at all. In those cases, formation flying of multiple spacecraft is the only logical configuration. In some cases, the position pattern required of the optical elements may negate the possibility of physically connecting them in any way. In other cases, the desire to degrade gracefully in the face of on-orbit problems may demand the kind of redundancy design that would not be possible with connected spacecraft. In still others, the sheer distances called for (i.e. LISA) would make even the idea of a tether too cumbersome to consider. Finally, in the cases where it is just not possible to use post-processing or direct optical pathlength control to allow natural motions of the structure (e.g., when the instrument or telescope design is prohibitive or optical correction scheme becomes overly complex), free flyers are the only choice. The advantage of the freeflying approach is simple. The total lack of rigidity of the synthetically-formed aperture is characterized by well-known dynamics among the rigid elements. A key challenge that is daunting to spacecraft engineers is the fact that wireless communication is required and control loops must be implemented through the communication links. However, the tremendous uncertainties of the flexible structure dynamics associated with deployable structures, booms, and tethers are eliminated in exchange for a requirement to develop actuation systems for rigid spacecraft as well as highly-precise metrology systems between the spacecraft.

\section{THE RIGHT ANSWER}

There is no silver bullet solution to the ultimate large aperture sensing design. Detailed analysis must be performed on a concept by concept basis. The selection should be made based on the following parameters:

1. optical design sensitivity analysis and error budget - the allowable displacements of the subapertures or sub-telescopes relative to one another in angle and translation 
2. the motions of the key performance points under a properly characterized external and internal disturbance environment (including self-induced disturbances caused by reaction wheels, path delay lines, etc)

3. the dynamic ranges and dynamic capability (bandwidth) of local correction schemes, such as fast steering mirrors, path delay lines, etc.

4. the required maximum baseline

5. the required orbit

6. the scientific requirement on vibrations in the image (power spectral density specification based on image quality).

The competition between the different approaches in a tradeoff should only occur when the requirements are on the borderline between the different approaches. For example, the line between the utility of using tethers and free-flyers can be very blurry. At the longer wavelengths in some configurations, the tethered approach may be the most sensible because the flexible effects may be below the requirements in the mid to long wave infrared. However, in very short wavelengths (short-wave infrared and below), the uncertain flexible dynamics may exhibit dynamic responses which are far beyond the capability of controlling by optical (e.g., with steering mirrors), passive or localized active means. Henceforth, the motions may be uncontrollable based on available actuation and may require unreasonable (i.e., overly complex, requiring too much power, and/or too much computational capability) amounts of control internal to the structure. The only solution left in this case would be a free-flying formation.

\section{REFERENCES}

1. Kailath, T. Linear Systems, pp. 120-175

2. L. Robertson, J. Leitner, J. Slater, B. de Blonk, "Integrated Modeling and Control of the UltraLITE System," Presented at the IEEE Aerospace Conference, Snowmass, CO., Feb. 1997.

3. M. Powers, et al., "Assessment of a Large Aperture Telescope Trade Space and Active OptoMechanical Control Architecture," Presented at the IEEE Aerospace Conference, Snowmass, CO., Feb. 1997.

4. Halverson, et al, Progress Towards Picometer Accuracy Metrology for the Space Interferometry Mission, International Conference of Space Optics, Dec. 2000, Toulouse, France.

5. Peterson, D. et al., The Space Interferometry Misson: Taking the Measure of the Universe, Final Report of the Space Interferometry Mission Science Working Group, Feb. 2000.

6. Teague, H., How, J. P., and Parkinson, B., "Attitude and Vibration Control of Flexible Structures Using GPS: Methods and Experimental Results", AIAA Journal Guidance, Control and Dynamics Sept-Oct, 1998, pp. 673-683.

7. Mather, J.C. et al., "The Submillimeter Frontier: A Space Science Imperative", 2000, Rev. Sci. Inst.

8. R. E.Farley, D.A.Quinn, "Tethered Formation Configurations: Meeting the Scientific Objectives of Large Aperture and Interferometric Science", Proceedings of the AIAA Space 2001 Conference \& Exposition Aug 2001. 